\title{
Innovation in Active Vibration Control Strategy of Intelligent Structures
}

\author{
A. Moutsopoulou, ${ }^{1}$ G. E. Stavroulakis, ${ }^{2}$ and A. Pouliezos ${ }^{2}$ \\ ${ }^{1}$ Department of Engineering (Civil), Technological Educational Institute of Crete, 71004 Heraklion, Crete, Greece \\ ${ }^{2}$ Department of Production Engineering and Management, Technical University of Crete, 73100 Chania, Crete, Greece \\ Correspondence should be addressed to A. Moutsopoulou; amalia@staff.teicrete.gr
}

Received 19 July 2013; Revised 13 November 2013; Accepted 14 November 2013; Published 6 March 2014

Academic Editor: Weihai Zhang

Copyright (c) 2014 A. Moutsopoulou et al. This is an open access article distributed under the Creative Commons Attribution License, which permits unrestricted use, distribution, and reproduction in any medium, provided the original work is properly cited.

Large amplitudes and attenuating vibration periods result in fatigue, instability, and poor structural performance. In light of past approaches in this field, this paper intends to discuss some innovative approaches in vibration control of intelligent structures, particularly in the case of structures with embedded piezoelectric materials. Control strategies are presented, such as the linear quadratic control theory, as well as more advanced theories, such as robust control theory. The paper presents sufficiently a recognizable advance in knowledge of active vibration control in intelligent structures.

\section{Introduction}

Modeling of an ideal system consisting of a beam with piezoelectric layers was done using one-dimensional finite elements with two degrees of freedom per node. Cubic and quadratic Hermit polynomials were employed for the representation of nodal rotations and vertical displacements. System differential equations are derived from Euler Bernoulli theory $[1,2]$.

Setting up the problem in a two-port diagram (inputoutput) was not a trivial task. The classic control problem was transformed into a two-port problem. The goal of nominal design was to keep error magnitude small, despite perturbations and noise in measurements. Moreover, controller size had to be contained so as to lower energy consumption and maintain piezoelectric materials within operating limits $( \pm 500 \mathrm{~V})$. By transforming transfer functions to state space equations and by using input and output equations, state space matrices have been derived; these matrices are used for finding the optimal controller according to the LQR and $H_{\infty}$ control criterion.

Selection of the weights involved in the controller we studied was done through optimization, while wind loading and noise in measurements were appropriately modelled for this particular problem. The obtained results were very satisfactory; beam vibration is reduced even for realistic wind measurements. Beam response results, with as well as without control, were compared for all presented control strategies.

In this paper, we address the problem of vibrations of intelligent structures. Stimuli may come from external perturbations of the system, disturbances, or excitation that may cause structural vibrations, such as wind loading or earthquakes. An intelligent structure is expected to be able to sense the vibration and counteract it in a controlled fashion, so that vibration of the system can be reduced and contained. To that end, a number of intelligent materials may be used as actuators and sensors. Piezoelectric materials, memory materials, and electrostrictive and magnetostrictive materials are such materials. In this work, we focus on the use of piezoelectric materials, given that they exhibit good sensing and actuation properties.

\section{Research on Intelligent Structures}

The following paragraphs give a deep insight into the research work done on the intelligent structures so far. Culshaw discussed the concept of smart structure, its benefits, and applications [3]. Rao and Sunar explained the use of piezo materials as sensors and actuators in sensing vibrations in 


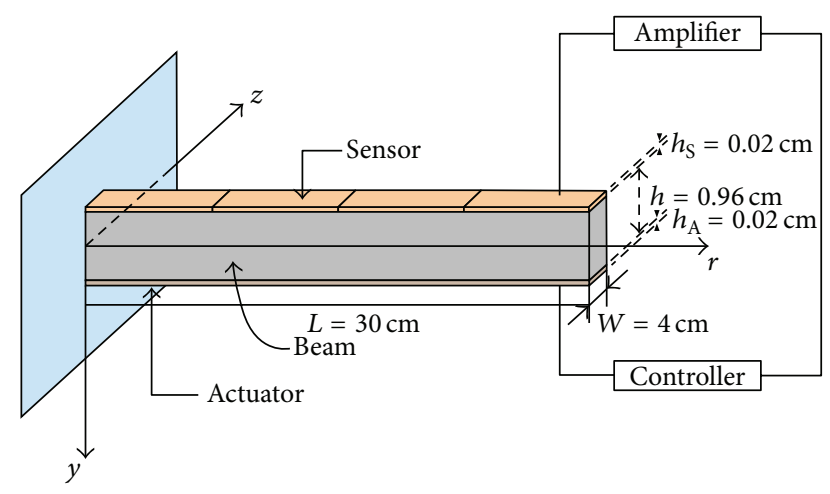

FIGURE 1: Smart beam.

their survey paper [4]. Hubbard and Baily have studied the application of piezoelectric materials as sensor/actuator for flexible structures [5]. Hanagud developed a Finite Element Model (FEM) for a beam with many distributed piezoceramic sensors/actuators [6].

Hwang and Park presented a new finite element (FE) modeling technique for flexible beams [7]. Continuous time and discrete time algorithms were proposed to control a thin piezoelectric structure by Bona et al. [8]. Schiehlen and Schonerstedt reported the optimal control designs for the first few vibration modes of a cantilever beam using piezoelectric sensors/actuators [9]. Choi have shown a design of position tracking sliding mode control for a smart structure [10]. Distributed controllers for flexible structures can be seen in Pourki [11].

A FEM approach was used by Benjeddou to model a sandwich beam with shear and extension piezoelectric elements [12]. The finite element model employed the displacement field of Zhang and Sun [13]. It was shown that the finite element results agree quite well with the analytical results. Raja et al. extended the finite element model of Benjeddou's research team to include a vibration control scheme [14].

\section{Mathematical Modeling}

A cantilever slender beam with rectangular cross-sections is considered. Four pairs of piezoelectric patches are embedded symmetrically at the top and the bottom surfaces of the beam, as shown in Figure 1.

The beam is from graphite-epoxy T300-976 and the piezoelectric patches are PZT G1195N. The top patches act like sensors and the bottom like actuators. The resulting composite beam is modelled by means of the classical laminated technical theory of bending. Let us assume that the mechanical properties of both the piezoelectric material and the host beam are independent in time. The thermal effects are considered to be negligible as well [15].

The beam has length $L$, width $W$, and thickness $h$. The sensors and the actuators have width $b_{\mathrm{S}}$ and $b_{\mathrm{A}}$ and thickness $h_{\mathrm{S}}$ and $h_{\mathrm{A}}$, respectively. The electromechanical parameters of the beam of interest are given in Table 1.
TABLE 1: Parameters of the composite beam.

\begin{tabular}{lc}
\hline Parameters & Values \\
\hline Beam length, $L$ & $0.3 \mathrm{~m}$ \\
Beam width, $W$ & $0.04 \mathrm{~m}$ \\
Beam thickness, $h$ & $0.0096 \mathrm{~m}$ \\
Beam density, $\rho$ & $1600 \mathrm{~kg} / \mathrm{m}^{3}$ \\
Young's modulus of the beam, $E$ & $1.5 \times 10^{11} \mathrm{~N} / \mathrm{m}^{2}$ \\
Piezoelectric constant, $d_{31}$ & $254 \times 10^{-12} \mathrm{~m} / \mathrm{V}$ \\
Electric constant, $\xi_{33}$ & $11.5 \times 10^{-3} \mathrm{~V} \mathrm{~m} / \mathrm{N}$ \\
Young's modulus of the piezoelectric element & $1.5 \times 10^{11} \mathrm{~N} / \mathrm{m}^{2}$ \\
Width of the piezoelectric element & $b_{\mathrm{S}}=b_{\mathrm{A}}=0.04 \mathrm{~m}$ \\
Thickness of the piezoelectric element & $h_{\mathrm{S}}=h_{\mathrm{A}}=0.0002 \mathrm{~m}$ \\
\hline
\end{tabular}

In order to derive the basic equations for piezoelectric sensors and actuators [1], we assume that

(i) the piezoelectric sensors actuators (S/A) are bonded perfectly on the host beam;

(ii) the piezoelectric layers are much thinner than the host beam;

(iii) the piezoelectric material is homogeneous, transversely isotropic, and linearly elastic;

(iv) the piezoelectric S/A are transversely polarized $[1,3$, 16].

3.1. Finite Element Formulation. We consider a beam element of length $L_{e}$, which has two mechanical degrees of freedom at each node: one translational $\omega_{1}$ (resp. $\omega_{2}$ ) in direction $z$ and one rotational $\psi_{1}$ (resp., $\psi_{2}$ ). The vector of nodal displacements and rotations $q_{e}$ is defined as [17]

$$
q_{e}^{r}=\left[\omega_{1}, \psi_{1}, \omega_{2}, \psi_{2}\right] .
$$

The beam element transverse deflection $\omega(x, t)$ and the beam element rotation $\psi(x, t)$ of the beam are continuous and they are interpolated within by Hermitian linear shape functions $H_{i}^{\omega}$ and $H_{i}^{\psi}$ as follows $[18,19]$ :

$$
\begin{aligned}
& \omega(x, t)=\sum_{i=1}^{4} H_{i}^{\omega}(x) q_{i}(t), \\
& \psi(x, t)=\sum_{i=1}^{4} H_{i}^{\psi}(x) q_{i}(t) .
\end{aligned}
$$

This classical finite element procedure leads to the approximate discretized variational problem. For a finite element, the discrete differential equations are obtained by substituting the discretized expressions into the first variation of the kinetic energy and strain energy $[18,20]$ to evaluate the kinetic and strain energies. Integrating over spatial domains and using the Hamiltons principle [20], the equation of motion for a beam element is expressed in terms of nodal variable $q$ as follows:

$$
M \ddot{q}(t)+D \dot{q}(t)+K q(t)=f_{m}(t)+f_{e}(t),
$$

where $M$ is the generalized mass matrix, $D$ the viscous damping matrix, $K$ the generalized stiffness matrix, $f_{m}$ 
the external loading vector, and $f_{e}$ the generalized control force vector, produced by electromechanical coupling effects. The independent variable $q(t)$ is composed of transversal deflections $\omega_{1}$ and rotations $\psi_{1}$; that is, $[16,18]$

$$
q(t)=\left[\begin{array}{c}
\omega_{1} \\
\psi_{1} \\
\vdots \\
\omega_{n} \\
\psi_{n}
\end{array}\right],
$$

where $n$ is the number of nodes used in analysis. Vectors $\omega$ and $f_{m}$ are positive upwards. To transform to state-space control representation, let (in the usual manner)

$$
\dot{x}(t)=\left[\begin{array}{l}
q(t) \\
\dot{q}(t)
\end{array}\right] .
$$

Furthermore, to express $f_{e}(t)$ as $B u(t)$, we write it as $f_{e}^{*} u$, where $f_{e}^{*}$ the piezoelectric force is for a unit applied on the corresponding actuator and $u$ represents the voltages on the actuators. Furthermore, $d(t)=f_{m}(t)$ is the disturbance vector $[16,18]$.

Then,

$$
\begin{aligned}
\dot{x}(t)= & {\left[\begin{array}{cc}
O_{2 n \times 2 n} & I_{2 n \times 2 n} \\
-M^{-1} K & -M^{-1} D
\end{array}\right] x(t) } \\
& +\left[\begin{array}{c}
O_{2 n \times 2 n} \\
M^{-1} f_{e}^{*}
\end{array}\right] u(t)+\left[\begin{array}{c}
O_{2 n \times 2 n} \\
M^{-1}
\end{array}\right] \\
= & A x(t)+B u(t)+G d(t) \\
= & A x(t)+[B \quad G]\left[\begin{array}{l}
u(t) \\
d(t)
\end{array}\right] \\
= & A x(t)+\widetilde{B} \widetilde{u}(t) .
\end{aligned}
$$

The previous description of the dynamical system will be augmented with the output equation (displacements only measured) [17] as follows:

$$
y(t)=\left[\begin{array}{llll}
x_{1}(t) & x_{3}(t) & \cdots & x_{n-1}(t)
\end{array}\right]^{T}=C x(t) .
$$

In this formulation, $u$ is $n \times 1$ (at most, but can be smaller), while $\mathrm{d}$ is $2 n \times 1$. The units used are compatible for instance $\mathrm{m}$, rad, sec, and $\mathrm{N}[21,22]$.

\section{Linear Quadratic Regulator: LQR Control}

It is well known $[23,24]$ that constant input disturbances can be eliminated only if the controller has a zero at infinity (i.e., it integrates). Another useful interpretation is that an integrator is a disturbance estimator. Hence we do not expect a zero steady-state error using an LQR controller.

The structure of LQR control with reduced order observer is shown in Figure 2.

Here, $d$ are the disturbances, $n$ is the noise, and the controller $K$ defines,

$$
K=\lim _{t \rightarrow \infty} K(t),
$$

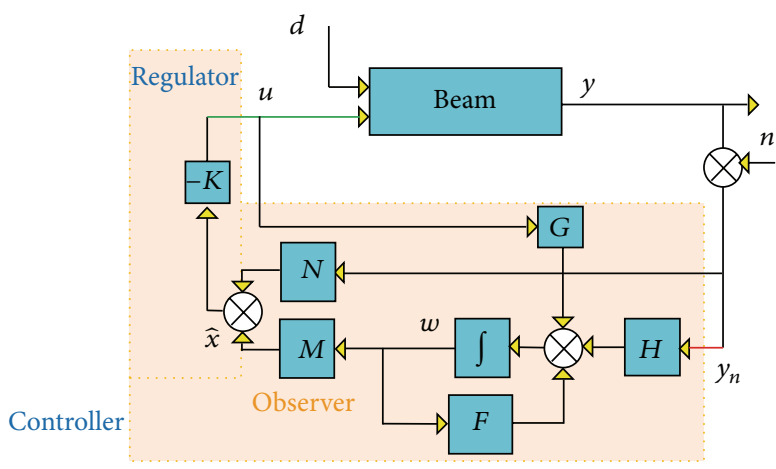

FIGURE 2: LQR controller with state estimator.

where

$$
u(t)=-K(t) \hat{x}(t)
$$

minimizes the weighted performance index as follows:

$$
J=\int_{0}^{\infty}\left(\hat{x}^{T}(t) Q \widehat{x}(t)+u^{T}(t) R u(t)\right) \mathrm{d} t
$$

and $Q$, and $R$ are design weight matrices.

The controller $K$ is given by relation

$$
K A-K B R^{-1} B^{T} K+Q+A^{T} K=0,
$$

which is the solution of the Riccati equation.

The weight matrices $Q$ and $R$ are used in order to:

(i) normalize the state and control vector;

(ii) assess the relative influence of deflection from equilibrium position and magnitude of control on the determination of a global criterion. Matrices $Q$ and $R$ are diagonal with positive diagonal inputs, so that

$$
\begin{aligned}
& \sqrt{Q_{i}}=\frac{1}{\max \left(x_{i}\right)}, \quad i=1,2, \ldots, m, \\
& \sqrt{R_{i}}=\frac{1}{\max \left(u_{i}\right)}, \quad i=1,2, \ldots, k .
\end{aligned}
$$

The value $\max \left(x_{i}\right)$ sets the maximum desirable output value $y$. The value $\max \left(u_{i}\right)$ has similar significance for input $u$.

Matrix $Q$ sets the weight for each state, while matrix $R$ holds the weight for each actuator's voltage. The LQR problem requires that the state be known [23].

\section{Inputs}

A typical wind load (Figure 3) Acting on the side of the structure. The wind load is a real-life wind speed measurement in relevance with time that took place in Estavromenos of Heraklion, Crete. We transform the wind speed in wind pressure. 


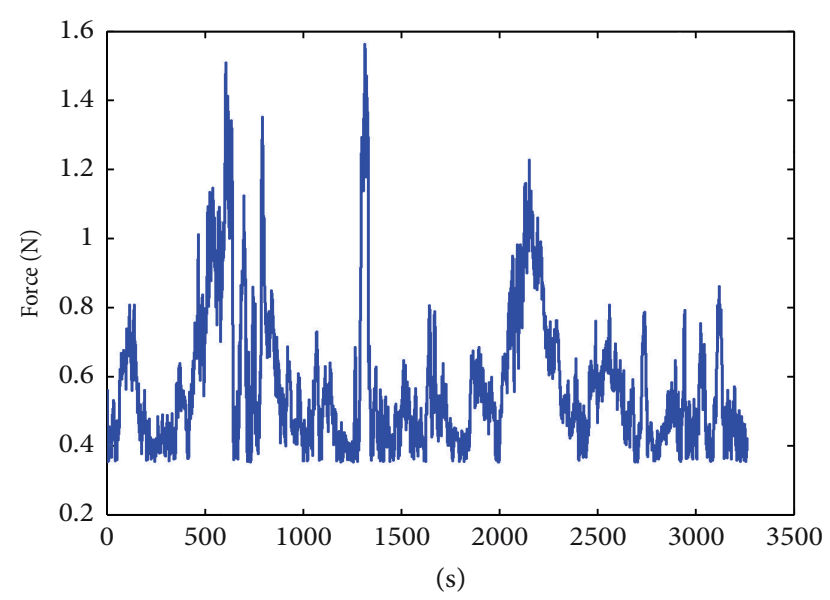

FIgURe 3: Wind load.

Loading corresponds to the wind excitation. The function $f_{m}(t)$ has been obtained from the wind velocity record, through the relation

$$
f_{m}(t)=\frac{1}{2} \rho C_{u} V^{2}(t),
$$

where $V=$ velocity, $\rho=$ density, and $C_{u}=1.5$.

Moreover, in all simulations, random noise has been introduced to measurements at system output locations within a probability interval of $\pm 1 \%$. Due to small displacements of system nodal points, noise amplitude is taken to be small, of the order of $5 \times 10^{-5}$. On the other hand, the signal is introduced at each node of the beam by a different percentage, that percentage being lower at the first node due to the fact that the beam end point is clamped.

\section{Results of Application of LQR Control}

The $Q$ and $R$ that were used are

$$
Q=100000 \times\left[\begin{array}{ccccccccc}
1 & 0 & 0 & 0 & 0 & 0 & 0 & \vdots & \\
0 & 0 & 0 & 0 & 0 & 0 & 0 & \vdots & \\
0 & 0 & 1 & 0 & 0 & 0 & 0 & \vdots & \\
0 & 0 & 0 & 0 & 0 & 0 & 0 & \vdots & 0_{7 \times 9} \\
0 & 0 & 0 & 0 & 1 & 0 & 0 & \vdots & \\
0 & 0 & 0 & 0 & 0 & 0 & 0 & \vdots & \\
0 & 0 & 0 & 0 & 0 & 0 & 1 & \vdots & \\
\cdots & \cdots & \cdots & \cdots & \cdots & \cdots & \cdots & \vdots & \cdots \\
& & & 0 & & & & & \\
& & & & & & & \\
9 \times 7 & & & & \vdots & 0_{9 \times 9}
\end{array}\right] .
$$

Since $\max \left(x_{i}\right)=0.00316228$ and $\max \left(u_{i}\right)=100$ (11), matrix $L$ is the design matrix. Its eigenvalues are chosen in such a way that the observer subsystem can be about two times faster than the observed system. The selected values for our simulation are

$$
\lambda_{L}=10^{7} \times\left[\begin{array}{c}
2.7423556 \\
-0.430498 \\
-0.031873 \\
-0.000051+0.0001993 i \\
-0.000051-0.000199 i \\
-0.00045+0.000053 i \\
-0.00045-0.000053 i \\
-0.00039+0.00001 i \\
-0.00039-0.00001 i \\
-0.0004 \\
-0.0004 \\
-0.0004
\end{array}\right]
$$

These values have been obtained by trial and error, given the poor numerical properties of the system. To find these values, we have used a robust pole computation algorithm included in MATLAB $[25,26]$.

The controller [Klqr] is given by relation (11) which is the solution of the Riccati equation, where $A$ and $B$ are respective state and control matrices of the system and $R$ and $Q$ are weight matrices of the performance criterion (regulator) (14) and (15), respectively.

For the simulation, beam nodal displacements and rotations with and without control are displayed in Figures 4 and 5, while Figure 6 presents actuator voltage values for control of all beam nodes.

6.1. Discussion of the Results of the Linear Quadratic Regulator $(L Q R)$. Using the linear quadratic controller criterion LQR, beam vibration reduction is observed at all nodal points, for both constant and sinusoidal mechanical input, as well as for realistic wind loading. LQR control achieves reduction of vibration but at the same time requires the entire system state time history as well as an extensive sensor distribution.

We encounter the following difficulties:

(i) system disturbances are unknown and unpredictable;

(ii) the state vector is not measurable in its entirety, which in turn necessitates the use of an observer. This setup is problematic, as the observer has no information on the disturbance, which results in erroneous estimates. A way to circumvent this problem is the use of an unknown input observer. Unfortunately, this approach is not feasible, as one of the prerequisite conditions is not met. This situation complicates the problem, making the application of classic controllers such as LQR difficult, since its performance is directly related to the availability of the state vector, or at best of a reliable estimator of the state vector.

For the reasons mentioned above, we will continue with a discussion of more advanced control techniques for this particular problem, such as the $H_{\infty}$ control. 


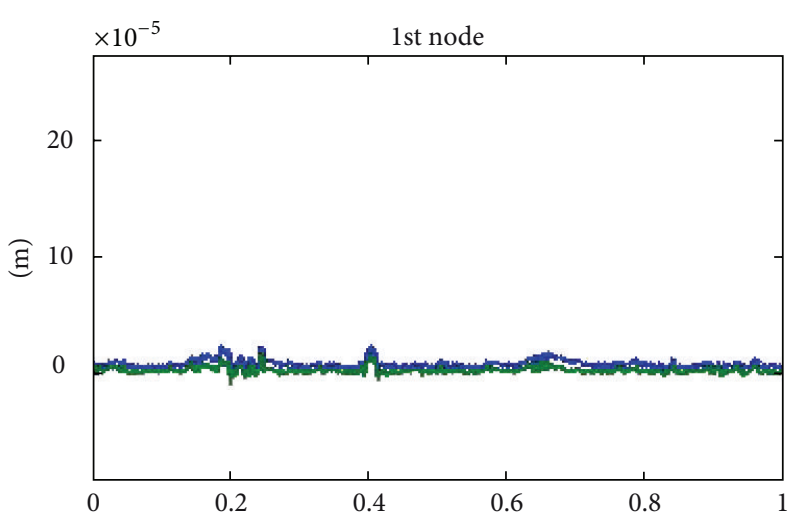

(s)

(a)

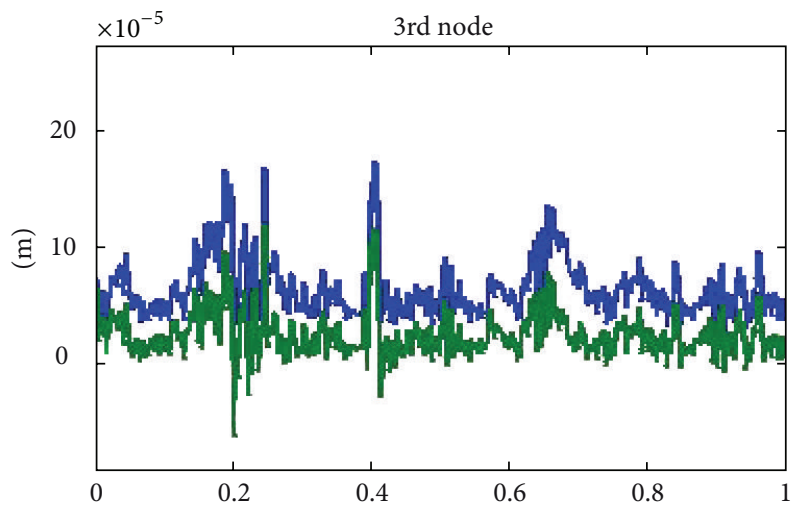

(s)

No control

LQR

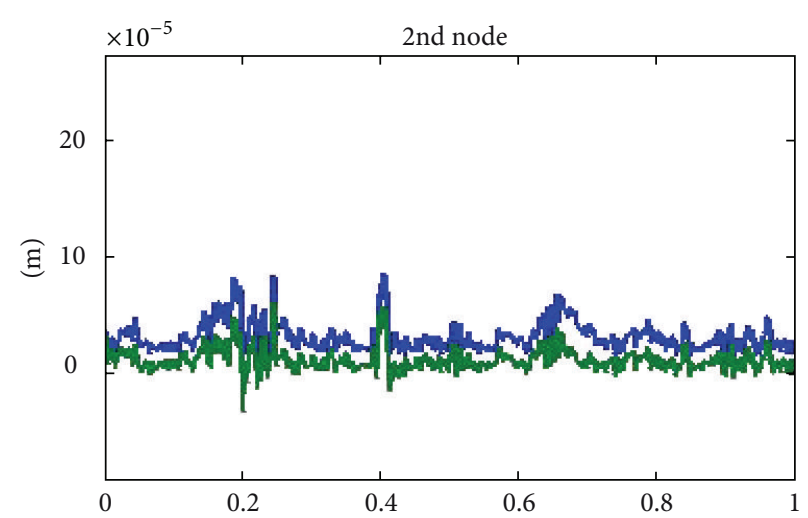

(s)

(b)

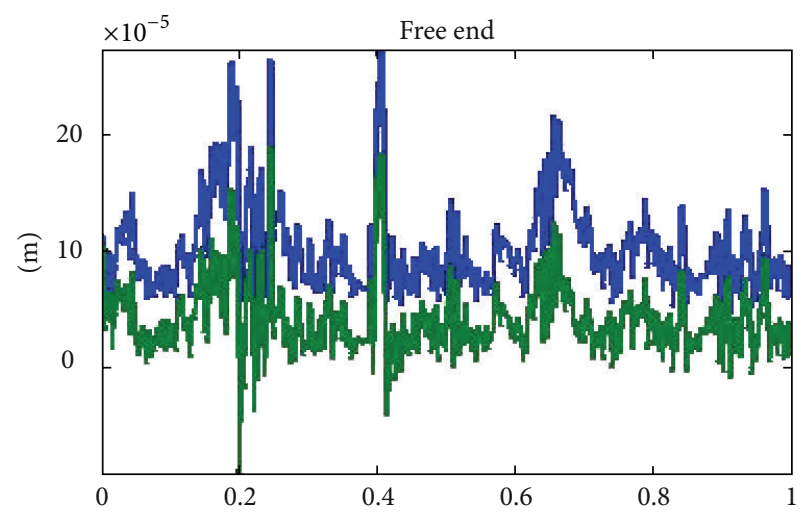

(s)

- No control
LQR

(c)

(d)

FIGURE 4: Displacement at all beam nodal points, with and without LQR control.

\section{7. $H_{\text {Infinity }}$ Control}

To relate the structures used in classical and $H_{\infty}$ control, let us look at Figure 7, in the frequency domain $[16,23,24]$.

In this diagram, all inputs and outputs of interest are included, along with their respective weighs $W$, where $W_{d}$, $W_{u}, W_{n}$, and $W_{y}$ are the weighs for the disturbances, control, noise, and outputs, respectively. The exogenous inputs are the noise $n$ and the disturbances $d . K(s)$ is the controller, $B, G, x$, $y, C$ define at the relation $(6,7,8)$, and $F(s)$ is the transfer function of our system.

To find the necessary transfer functions consider the following:

$$
\begin{aligned}
y_{F w} & =W_{y} J x=W_{y} J F v \\
& =W_{y} J F\left(G W_{d} d+B u_{K}\right) \\
& =W_{y} J F G W_{d} d+W_{y} J F B u_{K}, \\
u_{w} & =W_{u} u_{K},
\end{aligned}
$$

$$
\begin{aligned}
y_{n} & =C x+W_{n} n \\
& =C F v+W_{n} n \\
& =C F\left(G W_{d} d+B u_{K}\right)+W_{n} n \\
& =C F G W_{d} d+C F B u_{K}+W_{n} n .
\end{aligned}
$$

Combining all these gives

$$
\left[\begin{array}{c}
u_{w} \\
y_{F w} \\
\hline y_{n}
\end{array}\right]=\left[\begin{array}{cc|c}
0 & 0 & W_{u} \\
W_{y} J F G W_{d} & 0 & W_{y} J F B \\
\hline C F G W_{d} & W_{n} & C F B
\end{array}\right]\left[\begin{array}{c}
d \\
n \\
\hline u_{K}
\end{array}\right] .
$$

Note that the plant transfer function matrix, $F(s)$, is deduced from the suitably reformulated plant equations as follows:

$$
\begin{gathered}
\dot{x}(t)=A x(t)+I v(t), \\
y(t)=I x(t),
\end{gathered}
$$

where $v(t)=G d+B u_{k}$. 


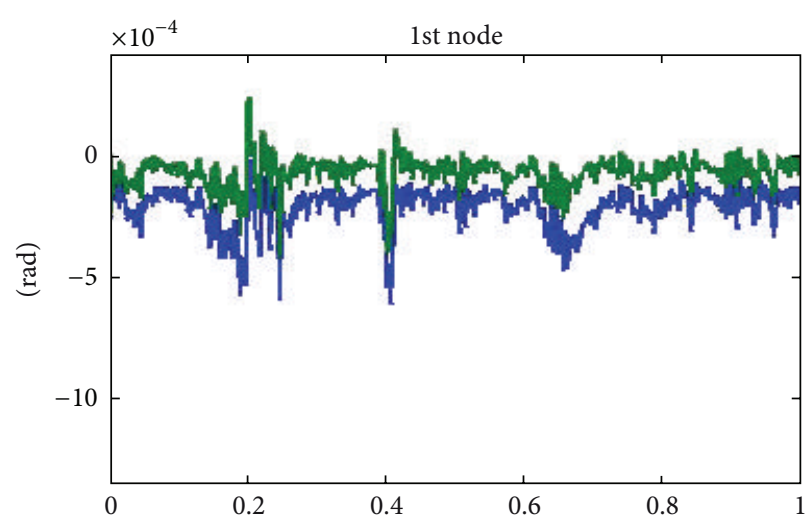

(a)

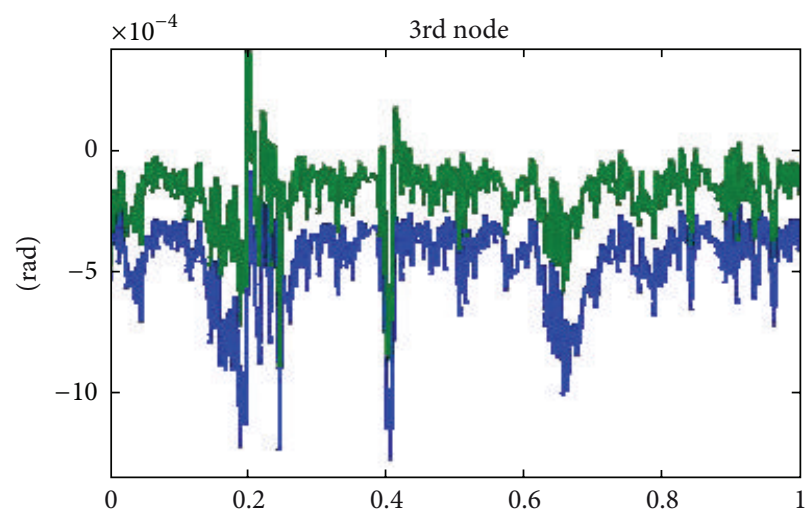

(c)

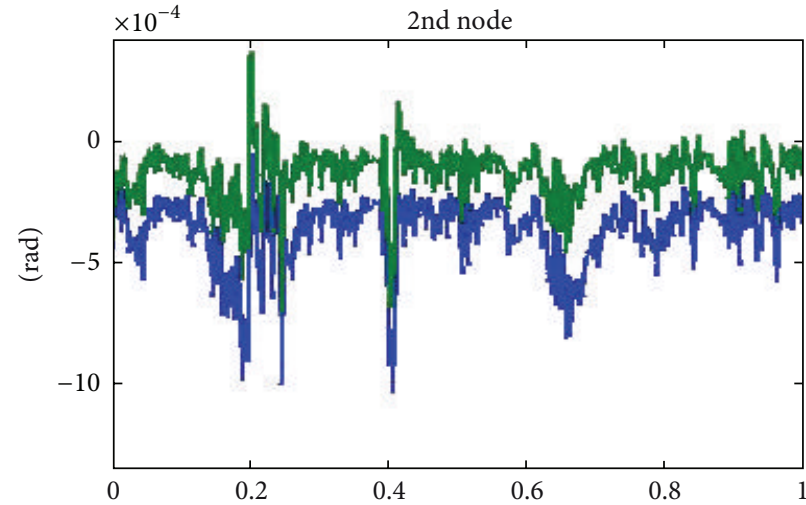

(b)

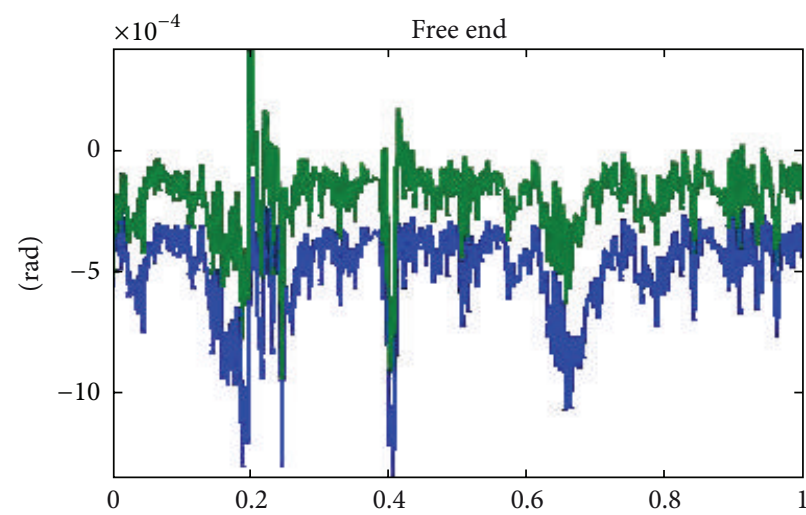

(d)

FIgURE 5: Angle of rotation at all beam nodal points, with and without LQR control.

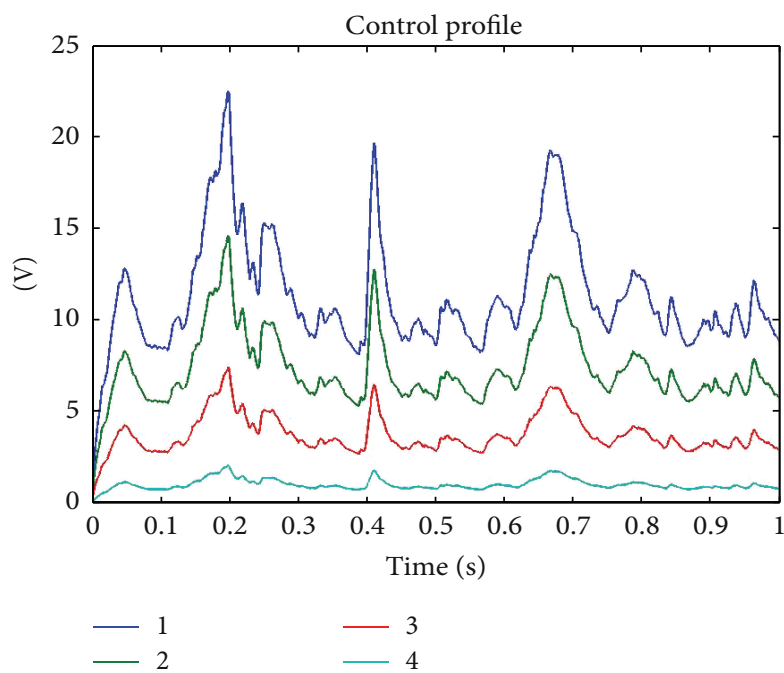

FIGURE 6: Control produced voltage at all beam nodal points with LQR control.

Hence,

$$
F(s)=(s I-A)^{-1}
$$

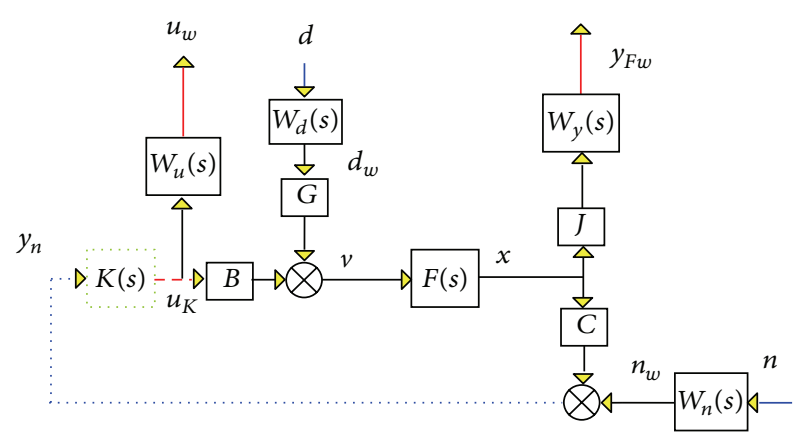

FIGURE 7: $H_{\text {Infinity }}$ control bloc diagram in the frequency domain.

The equivalent two-port diagram in the state space form is shown in Figure 8 for the close loop, and with more details in Figure 9, with

$$
z=\left[\begin{array}{c}
u_{w} \\
y_{F w}
\end{array}\right], \quad w=\left[\begin{array}{l}
d \\
n
\end{array}\right], \quad y=y_{n}, \quad u=u_{K},
$$

where $z$ are the output variables to be controlled and $w$ the exogenous inputs. 


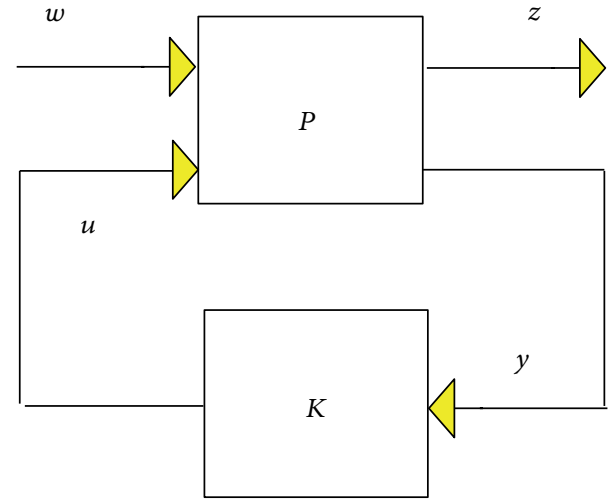

Figure 8: Two-port diagram.

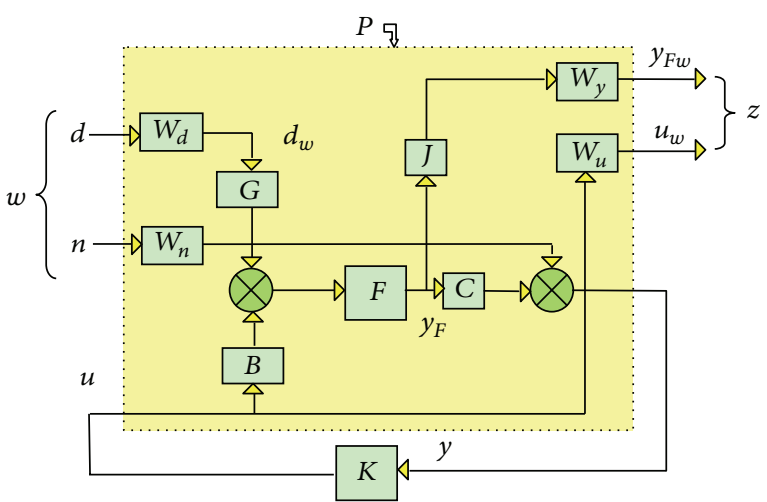

Figure 9: Details of $H_{\infty}$ structure.

Given that $P$ has two inputs and two outputs, it is, as usual, naturally partitioned as

$$
\left[\begin{array}{l}
z(s) \\
y(s)
\end{array}\right]=\left[\begin{array}{lll}
P_{z w}(s) & P_{z u}(s) \\
P_{y w}(s) & P_{y u}(s)
\end{array}\right]\left[\begin{array}{l}
w(s) \\
u(s)
\end{array}\right] \stackrel{\mathrm{o} \rho}{=} P(s)\left[\begin{array}{l}
w(s) \\
u(s)
\end{array}\right] .
$$

Also,

$$
u(s)=K(s) y(s) .
$$

Using (18) the transfer function for $P$ which is

$$
P(s)=\left[\begin{array}{cc|c}
0 & 0 & W_{u} \\
W_{y} J F G W_{d} & 0 & W_{y} J F B \\
\hline C F G W_{d} & W_{n} & C F B
\end{array}\right],
$$

while the closed loop transfer function for $M_{z w}(s)$ is

$$
M_{z w}(s)=P_{z w}(s)+P_{z u}(s) K(s)\left(I-P_{y u}(s) K(s)\right)^{-1} P_{y w}(s) \text {, }
$$

or

$$
z=M_{z w} w=F_{l}(P, K) w .
$$

Equation (25) is the well known lower LFT for $M_{z w}$.

To express $P$ in state space form, the natural partitioning

$$
P(s)=\left[\begin{array}{c|cc}
A & B_{1} & B_{2} \\
\hline C_{1} & D_{11} & D_{12} \\
C_{2} & D_{21} & D_{22}
\end{array}\right]=\left[\begin{array}{ccc}
P_{z w}(s) & P_{z u}(s) \\
P_{y w}(s) & P_{y u}(s)
\end{array}\right]
$$

is used (where the packed form has been used), while the corresponding form for the controller $K$ is [27-29]

$$
K(s)=\left[\begin{array}{l|l}
A_{K} & B_{K} \\
\hline C_{K} & D_{K}
\end{array}\right] .
$$

Equation (27) defines the following equations:

$$
\begin{gathered}
\dot{x}(t)=A x(t)+\left[\begin{array}{ll}
B_{1} & B_{2}
\end{array}\right]\left[\begin{array}{l}
w(t) \\
u(t)
\end{array}\right], \\
{\left[\begin{array}{l}
z(t) \\
y(t)
\end{array}\right]=\left[\begin{array}{l}
C_{1} \\
C_{2}
\end{array}\right] x(t)+\left[\begin{array}{ll}
D_{11} & D_{12} \\
D_{21} & D_{22}
\end{array}\right]\left[\begin{array}{l}
w(t) \\
u(t)
\end{array}\right],} \\
\dot{x}_{K}(t)=A_{K} x_{K}(t)+B_{K} y(t), \\
u(t)=C_{K} x_{K}(t)+D_{K} y(t) .
\end{gathered}
$$

To find the matrices involved, we break the feedback loop and use the relevant equations.

Therefore the equations relating the inputs, outputs, states, and input/output to the controller are

$$
\begin{gathered}
\dot{x}_{F}=A x_{F}+\left(G d_{w}+B u\right), \quad y_{F}=x_{F}, \\
\dot{x}_{u}=A_{u} x_{u}+B_{u} u, \quad u_{w}=C_{u} x_{u}+D_{u} u, \\
\dot{x}_{y F}=A_{y F} x_{y F}+B_{y F} J y_{F}, \quad y_{F w}=C_{y F} x_{y F}+D_{y F} y_{F}, \\
\dot{x}_{n}=A_{n} x_{n}+B_{n} n, \quad n_{w}=C_{n} x_{n}+D_{n} n, \\
\dot{x}_{d}=A_{d} x_{d}+G d, \quad d_{w}=C_{d} x_{d}+D_{d} d, \\
y_{n}=C y_{F}+n_{w}, \\
x=\left[\begin{array}{c}
x_{F} \\
x_{u} \\
y_{F w} \\
x_{n} \\
x_{d}
\end{array}\right], \quad \begin{array}{c}
y=y_{n}, \\
z=\left[\begin{array}{c}
u_{w} \\
y_{F w}
\end{array}\right], \quad u=u_{K} .
\end{array}
\end{gathered}
$$

From (30), we use $d_{w}, n_{w} \kappa \alpha \iota y_{F w}$ and take our initial state space equation in the form of $(6,7,8)$, as follows:

$$
\begin{aligned}
& \dot{x}=\left[\begin{array}{ccccc}
A_{G} & 0 & 0 & 0 & G C_{d} \\
0 & A_{u} & 0 & 0 & 0 \\
B C_{F} & 0 & A_{y F} & 0 & 0 \\
0 & 0 & 0 & A_{n} & 0 \\
0 & 0 & 0 & 0 & A_{d}
\end{array}\right] x \\
& +\left[\begin{array}{cc}
G D_{d} & 0 \\
0 & 0 \\
0 & 0 \\
0 & B_{n} \\
B_{d} & 0
\end{array}\right] w+\left[\begin{array}{c}
B \\
B_{u} \\
0 \\
0 \\
0
\end{array}\right] u, \\
& z=\left[\begin{array}{ccccc}
0 & C_{u} & 0 & 0 & 0 \\
D_{y F} C_{F} & 0 & C_{y F} & 0 & 0
\end{array}\right] x+0 w+\left[\begin{array}{c}
D_{u} \\
0
\end{array}\right] u \text {, } \\
& y=\left[\begin{array}{lllll}
C_{F} & 0 & 0 & C_{n} & 0
\end{array}\right] x+\left[\begin{array}{ll}
0 & D_{n}
\end{array}\right] w+0 u .
\end{aligned}
$$




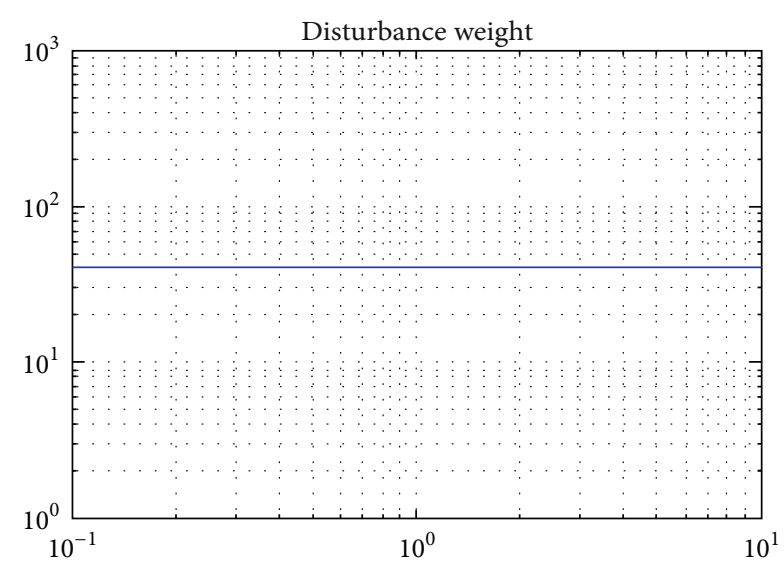

(a)

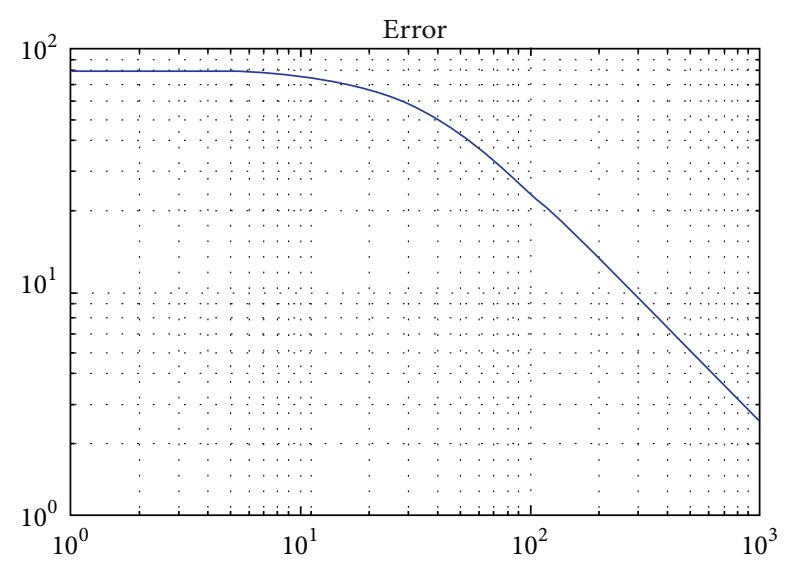

(c)

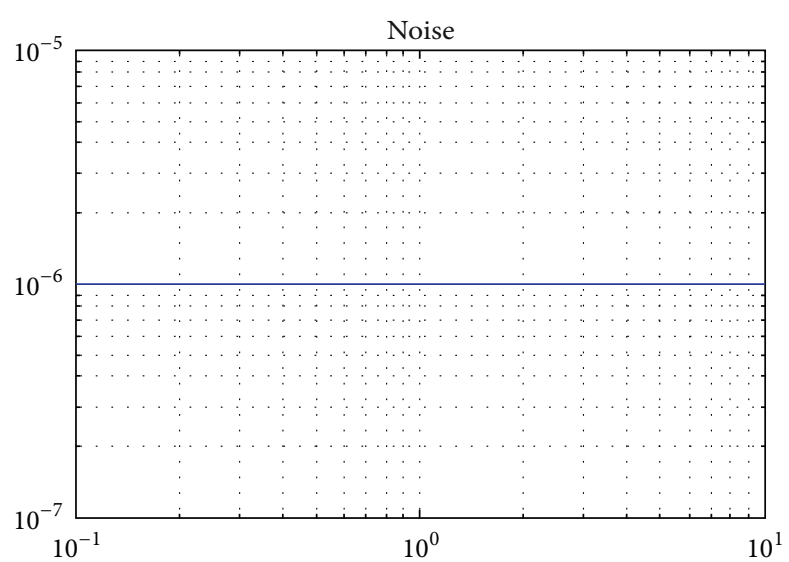

(b)

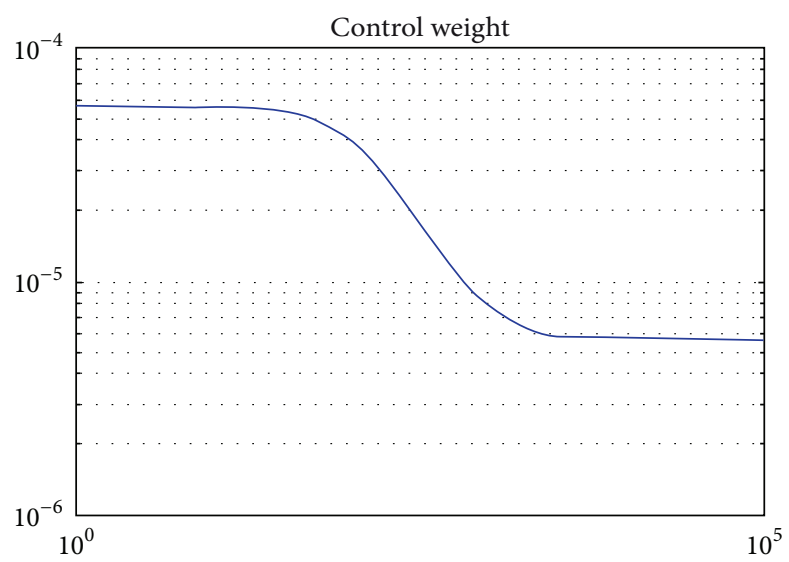

(d)

FIGURE 10: Bode diagrams of diagonal elements of weight matrices.

Therefore the matrices are

$$
\begin{aligned}
& A_{1}=\left[\begin{array}{ccccc}
A_{F} & 0 & 0 & 0 & G C_{d} \\
0 & A_{u} & 0 & 0 & 0 \\
B C_{F} & 0 & A_{y F} & 0 & 0 \\
0 & 0 & 0 & A_{n} & 0 \\
0 & 0 & 0 & 0 & A_{d}
\end{array}\right], \\
& B_{1}=\left[\begin{array}{cc}
G D_{d} & 0 \\
0 & 0 \\
0 & 0 \\
0 & B_{n} \\
B_{d} & 0
\end{array}\right], \quad B_{2}=\left[\begin{array}{c}
B \\
B_{u} \\
0 \\
0 \\
0
\end{array}\right] \text {, } \\
& C_{1}=\left[\begin{array}{ccccc}
0 & C_{u} & 0 & 0 & 0 \\
D_{y F} C_{F} & 0 & C_{y F} & 0 & 0
\end{array}\right] \text {, } \\
& D_{11}=0, \quad D_{12}=\left[\begin{array}{c}
D_{u} \\
0
\end{array}\right], \\
& C_{2}=\left[\begin{array}{lllll}
C_{F} & 0 & 0 & C_{n} & 0
\end{array}\right], \quad D_{21}=\left[\begin{array}{ll}
0 & D_{n}
\end{array}\right], \quad D_{22}=0 \text {. }
\end{aligned}
$$

7.1. Results with $H_{\infty}$. Figure 10 presents the Bode diagrams of diagonal elements of the above weight matrices. These matrices have been obtained through a number of tests, to ensure the feasibility of finding a controller $H_{\infty}$.

The controller obtained by applying $H_{\infty}$ control is 36 order. For this controller, $\gamma=0.074$. A plot of the maximum singular value of the weighted closed loop system (beam plus $H_{\infty}$ controller) is given in Figure 11, where we can clearly note that the value remains below $\gamma$ at all frequencies.

Figures 12, 13, and 14 further show the maximum singular values of transfer functions of the unweighted closed loop system (i.e., the initial one) that are of interest.

These figures show that the performance of the computed controller is satisfactory [30] since:

(i) as shown in Figure 12, there is a significant improvement in the effect of disturbance on error up to the frequency of $1000 \mathrm{~Hz}$;

(ii) as shown in Figure 13, there seems to be little effect of noise on error for frequencies beyond $1000 \mathrm{~Hz}$;

(iii) Figure 14 shows a satisfactory effect of the disturbance on the size of the control scheme (the design could be improved, if it were possible to reduce noise effect for frequencies of $1000 \mathrm{~Hz}$ ). 
$H_{\infty}$ control unweighted closed loop: max. singular value

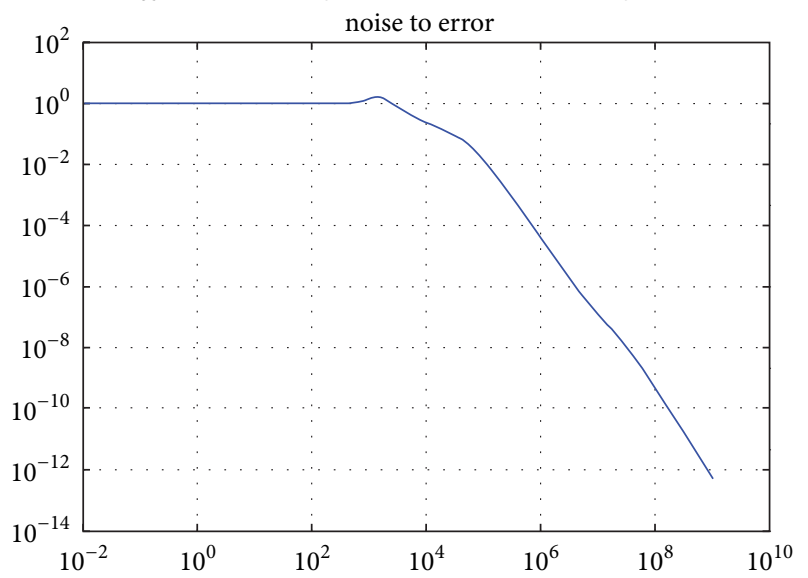

FIGURE 11: Maximum singular value of the unweighted closed loop system.

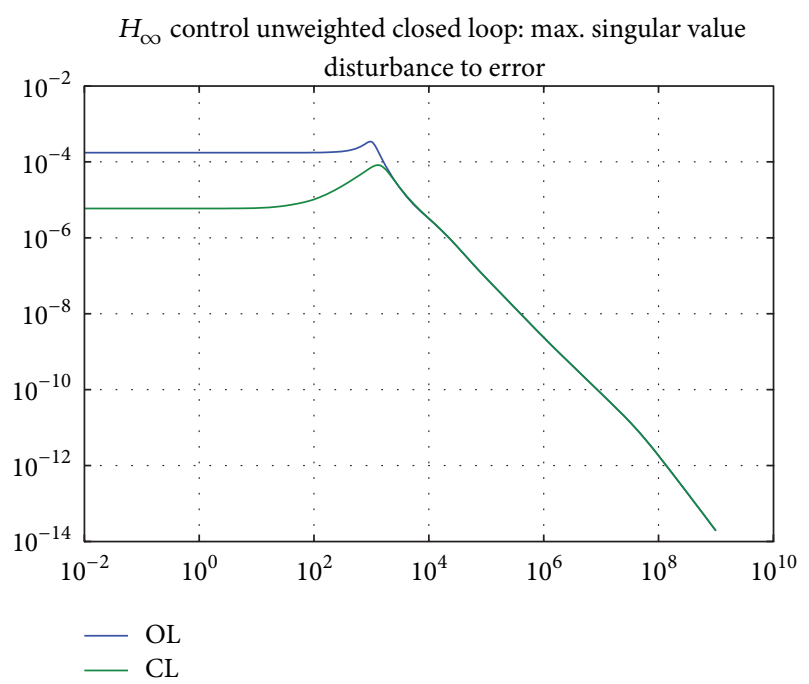

FIgURE 12: Maximum singular value disturbance to error.

To validate the above findings, system response time histories for the three input cases mentioned in this section are presented below.

Using the mechanical input, we get the following result.

Figure 15 shows the displacement time history at all nodal points of the beam, with and without control, while Figure 16 displays the angle of rotation time history at all beam nodal points, with and without control. By employing the $H_{\infty}$ control, vibration reduction is achieved; we observe vibration reduction of $90 \%$. Figure 17 presents the time evolution of the produced actuator voltage, which turns out to be lower than the piezoelectric voltage limit value of $500 \mathrm{~V}$.

7.2. Order Reduction of Controller $H_{\infty}$. The $H_{\infty}$ controller found is of order 36 . The fact that controller order, which is equal to the order of the system, is relatively higher than the order of classical controllers such as PI and LQR has led a number of researchers to develop order reduction algorithms.

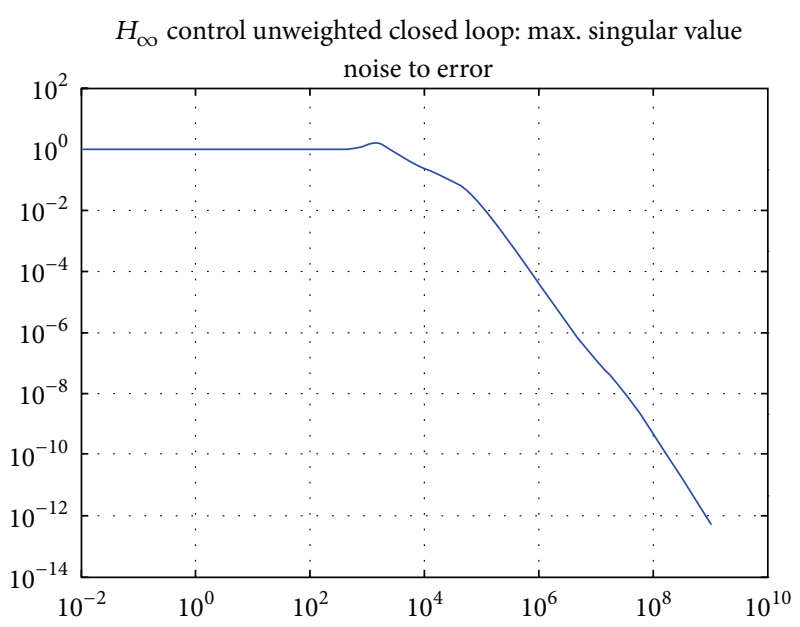

FIgURE 13: Maximum singular value noise to error.

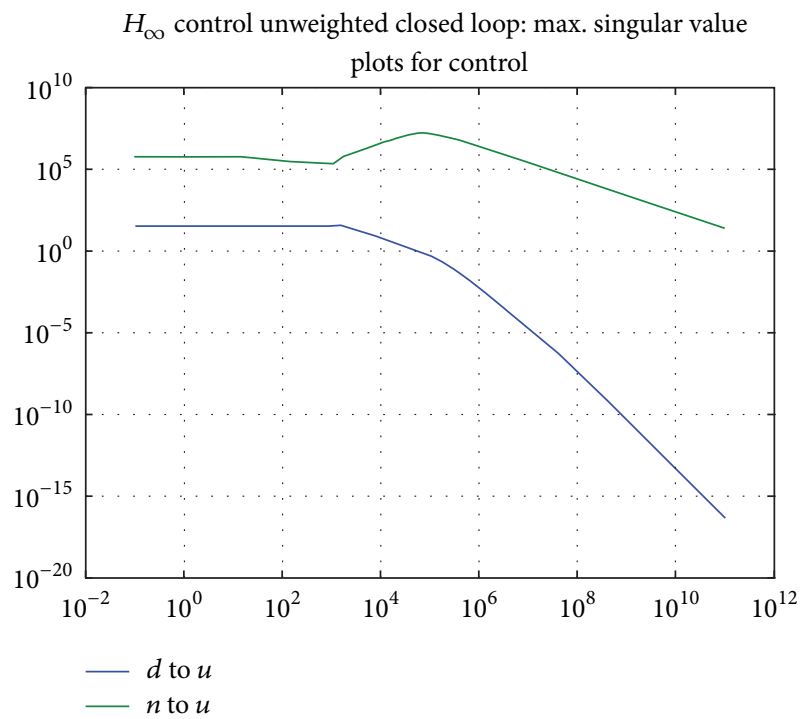

FIGURE 14: Maximum singular value plots for control.

The most widely used such algorithm, known as HIFOO, has been implemented in a Matlab environment, and is the one used in the following procedure [31].

The general problem is to compute a controller of reduced rank/order $n<36$ while retaining the performance of the $H_{\infty}$ criterion as well as the behaviour of a full order controller for the given system $[32,33]$ as follows:

$$
\begin{gathered}
\dot{x}(t)=A x(t)+B_{1} w(t)+B_{2} u(t), \\
z(t)=C_{1} x(t)+D_{11} w(t)+D_{12} u(t), \\
y(t)=C_{2} x(t)+D_{21} w(t)+D_{22} u(t) .
\end{gathered}
$$

The state space equations for the controller $K$ are

$$
\begin{gathered}
\dot{x}_{K}(t)=A_{K}(t)+B_{K} y(t), \\
u(t)=C_{K}(t)+D_{K} y(t) .
\end{gathered}
$$




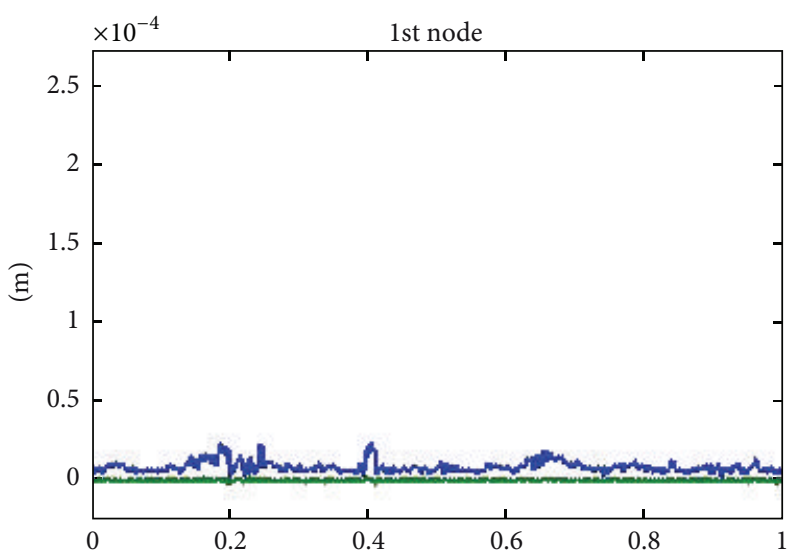

(s)

(a)

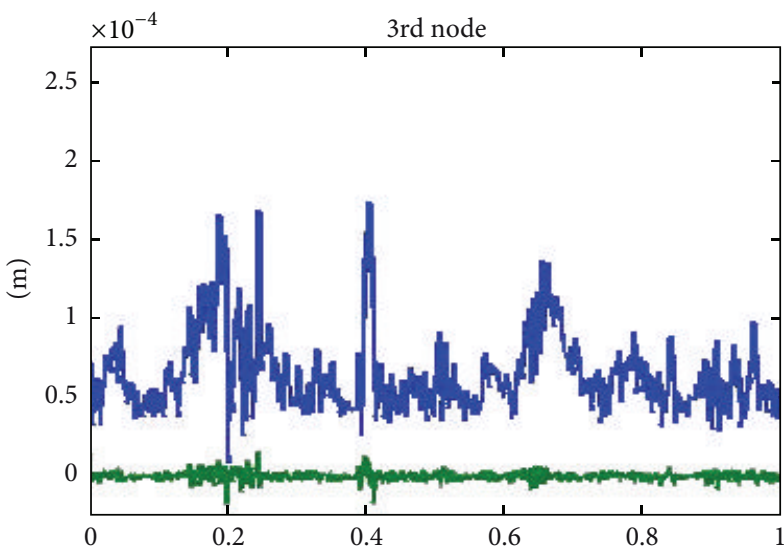

(s)

No control
Hinf

(c)

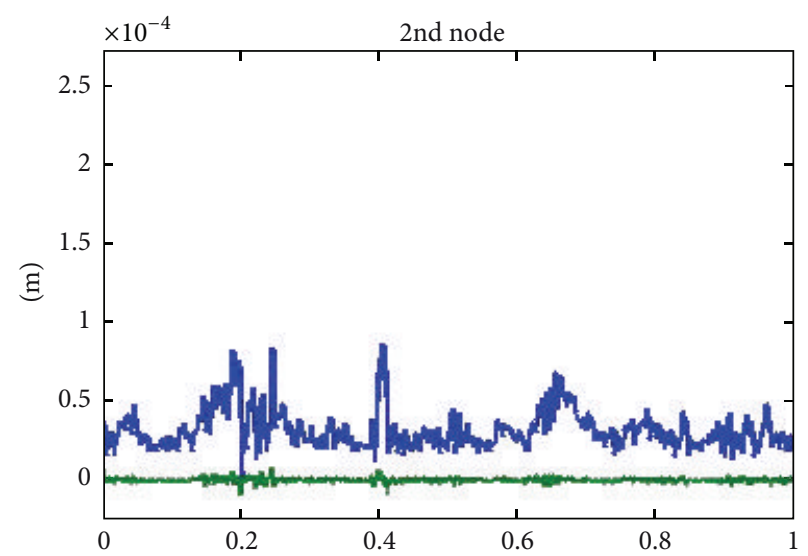

$(\mathrm{s})$

(b)

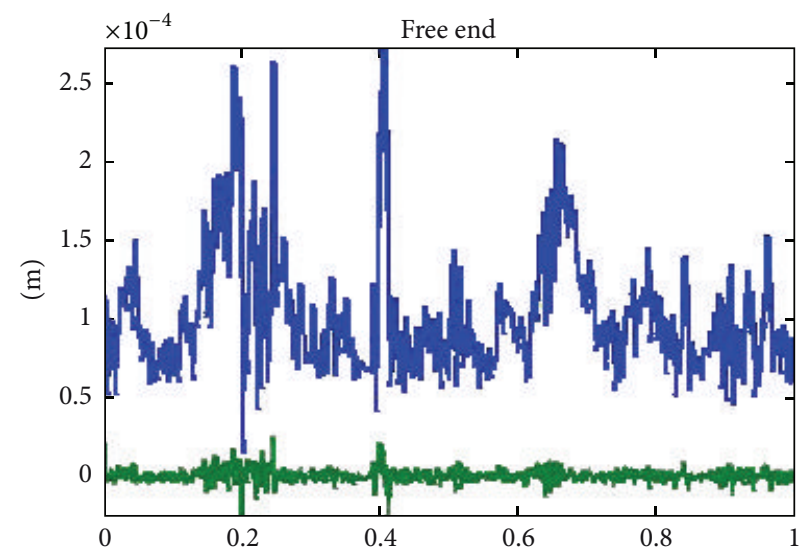

(s)

- No control

- Hinf

FIGURE 15: Displacement at all beam nodal points, with and without $H_{\infty}$ control.

Let $\alpha(X)$ be the spectral abscissa of a matrix $X$, that is, the maximum real part of its eigenvalues. Then, we require not only that $\alpha\left(A_{\mathrm{CL}}\right)<0$, where $A_{\mathrm{CL}}$ is the closed loop system matrix, but that $\alpha\left(A_{k}\right)<0$ as well. The feasible set of $A_{k}$, that is the set of stable matrices, is not a convex set and has a boundary that is not smooth $[34,35]$.

The HIFOO procedure has two phases: stability and performance optimization $[31,36]$. In the stability phase, HIFOO attempts to minimize

$$
\max \left(\alpha\left(A_{\mathrm{CL}}, \in \alpha\left(A_{\mathrm{CL}}\right)\right)\right),
$$

where $\varepsilon$ is a positive parameter that will be described shortly, until a controller is found for which this quantity is negative; that is, the controller is stable and makes the closed loop system stable. In case it is unable to find such a controller, HIFOO terminates unsuccessfully.
In the performance optimization phase, HIFOO searches for a local minimizer of

$f(K)$

$= \begin{cases}\infty, & \text { if } \max \left(\alpha\left(A_{\mathrm{CL}}, \alpha\left(A_{K}\right)\right)\right) \geq 0, \\ \max \left(\left\|T_{z w}\right\|_{\infty}, \in\|K\|_{\infty}\right), & \text { if else, }\end{cases}$

where

$$
\|\mathrm{K}\|_{\infty}=\sup _{R s=0}\left\|C_{k}\left(s I-A_{k}\right)^{-1} B_{K}+D_{K}\right\|_{2} .
$$

The introduction of $\varepsilon$ is motivated by the fact that the main design objective is to attain closed loop system stability and to minimize $\left\|T_{z w}\right\|_{\infty}$, by demonstrating that $\varepsilon$ should be relatively small; the term $\varepsilon\|K\|_{\infty}$, however, prevents the controller $H_{\infty}$ norm from becoming too large, in which case the stability constraint by itself would not exist. Given 


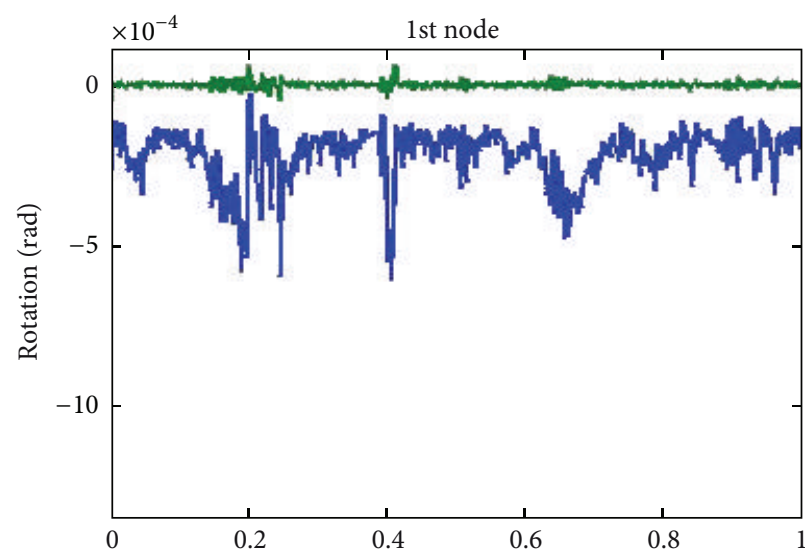

(s)

(a)

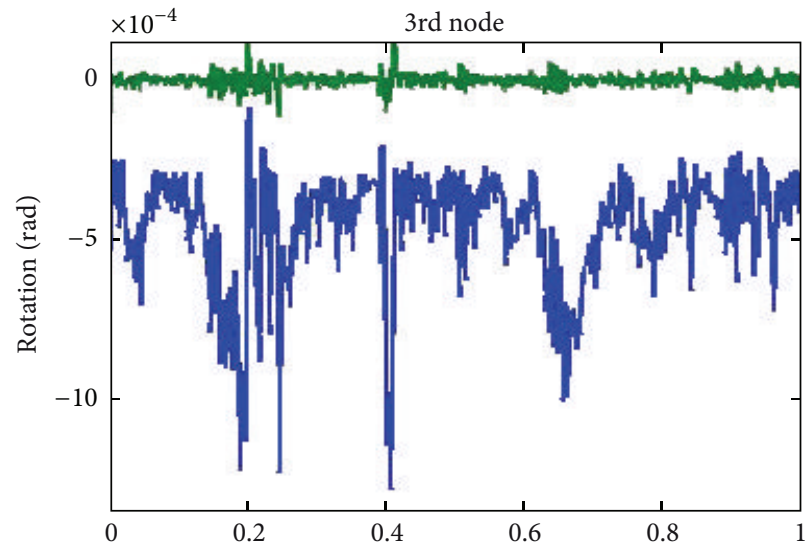

(s)

(c)

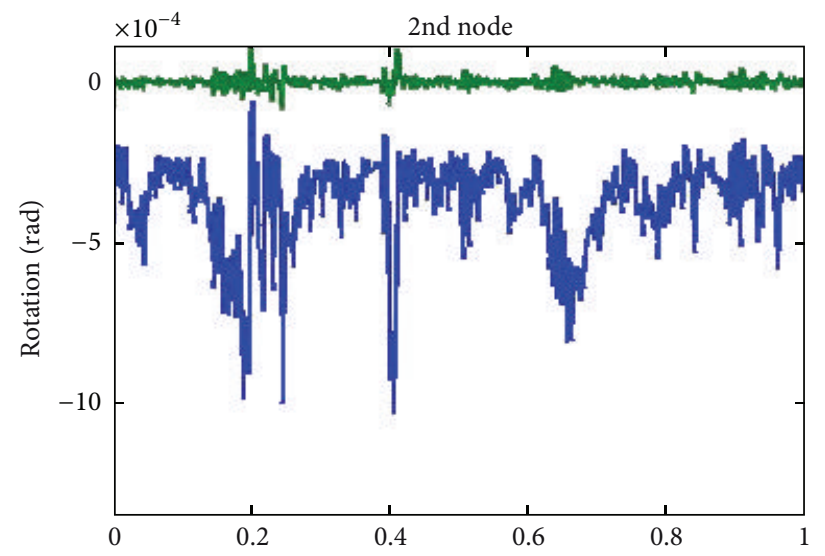

(s)

(b)

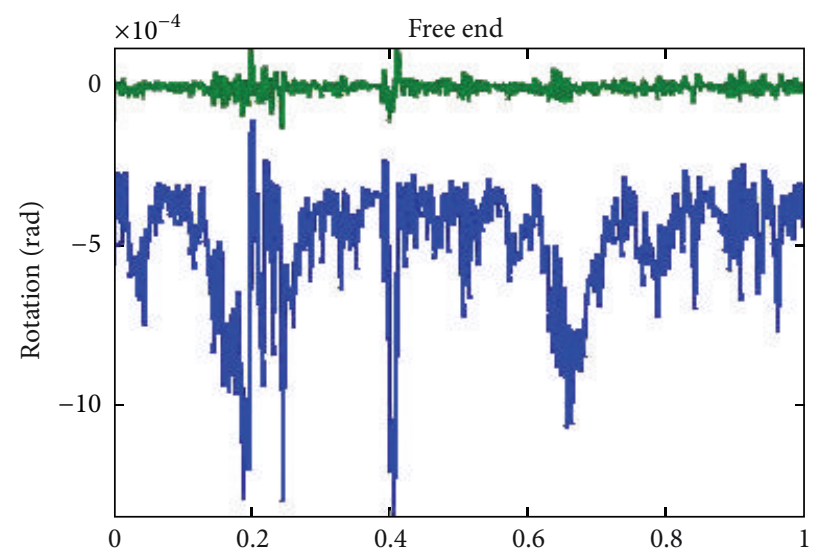

(s)

(d)

FIGURE 16: Angle of rotation at all beam nodal points, with and without $H_{\infty}$ control.

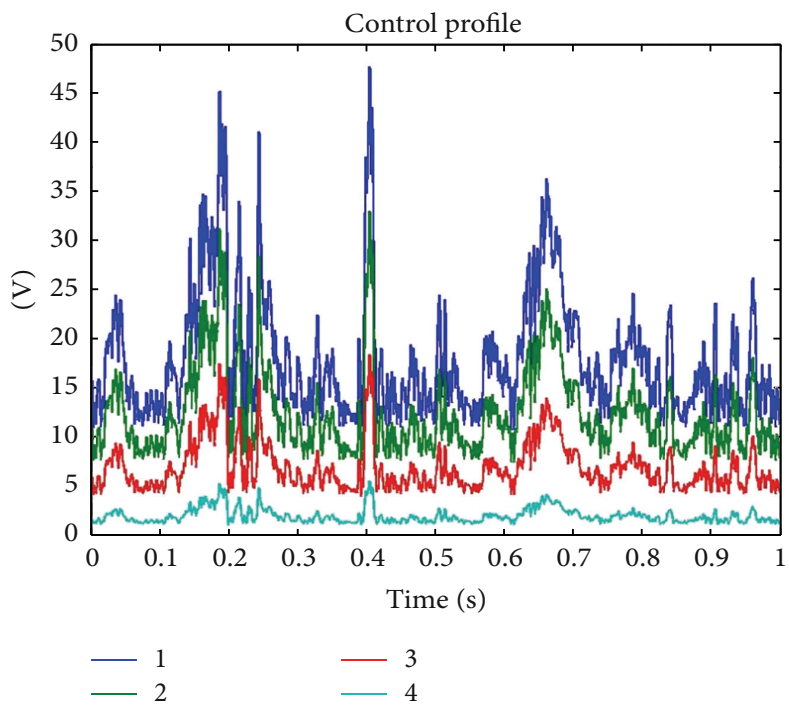

FIGURE 17: Control produced voltage at all beam nodal points with $H_{\infty}$ control. 
that it is preceded by the stability phase, the performance optimization phase is initialized with a finite value of $f(K)$. Consequently, when it reaches a value of $K$ for which $f(K)=$ $\infty$, that value is rejected, since an objective reduction is sought at each iteration $[31,36]$.

7.3. Results Using Controller HIFOO. As mentioned before, the HIFOO controller is implemented in Matlab by way of appropriate routines. It is called in the following manner:

$$
\mathrm{Kfoo}=\text { hifoo }(\text { plant, } 2),
$$

where plant is the system description in the form of (33) and $n=2$ is the controller order.

The resulting controller is described in state space in similar manner as $H_{\infty}$; that is,

$$
\begin{gathered}
\dot{x}_{K}(t)=A_{K}(t)+B_{K} y(t), \\
u(t)=C_{K}(t)+D_{K} y(t) .
\end{gathered}
$$

The controller state space equation is given by (39), where controller matrices are equal to

$$
\begin{gathered}
A_{K}=\left[\begin{array}{ll}
728.1 & -5034 \\
207.5 & -1408
\end{array}\right], \\
B_{K}=\left[\begin{array}{cccc}
212.8 & 811.6 & 1716 & 2810 \\
-164.9 & -637.2 & -1348 & -2207
\end{array}\right], \\
C_{K}=\left[\begin{array}{ccc}
1557 & -916.7 \\
1013 & -592.3 \\
517 & -297.9 \\
144.3 & -82.59
\end{array}\right] \\
D_{K}=\left[\begin{array}{cccc}
36.1 & 136.6 & 287.1 & 468.3 \\
23.5 & 87.69 & 186.5 & 303 \\
12.12 & 44.12 & 93.39 & 154.3 \\
4.204 & 12.53 & 26.92 & 43.51
\end{array}\right] .
\end{gathered}
$$

For the purpose of comparison of HIFOO controller performance to that of $H_{\infty}$, the beam free end response is examined, for the mechanical input.

For the input in Figure 18, the beam free end response is shown, initially with and then without the HIFOO controller, while Figure 19 presents produced actuator voltage using the HIFOO controller.

Using the HIFOO controller for an actual wind loading, beam position control is effected with node displacements of order of $10^{-5}$, with lower produced voltage. We therefore maintain $H_{\infty}$ criterion performance with a lower order controller. The maximum produced voltage for the HIFOO controller is $7 \mathrm{~V}$; the respective value is $45 \mathrm{~V}$ for the $H_{\infty}$ controller. In other words, beam adjustment to its equilibrium position is achieved with a lower order controller that requires lower voltage; see Figure 19.

\section{Results}

In the present work, the use of active control technology in intelligent structures has been presented. The goal of control

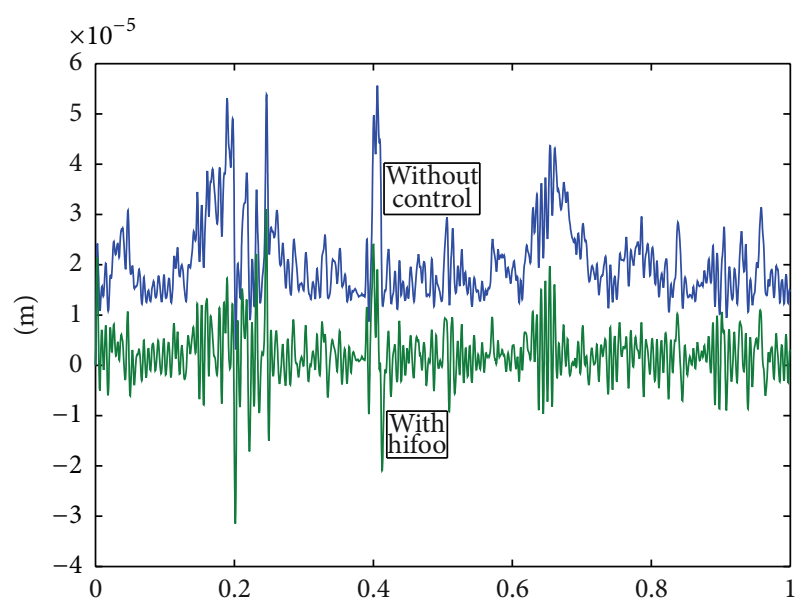

(s)

FIGURE 18: Beam free end displacement, with and without HIFOO control.

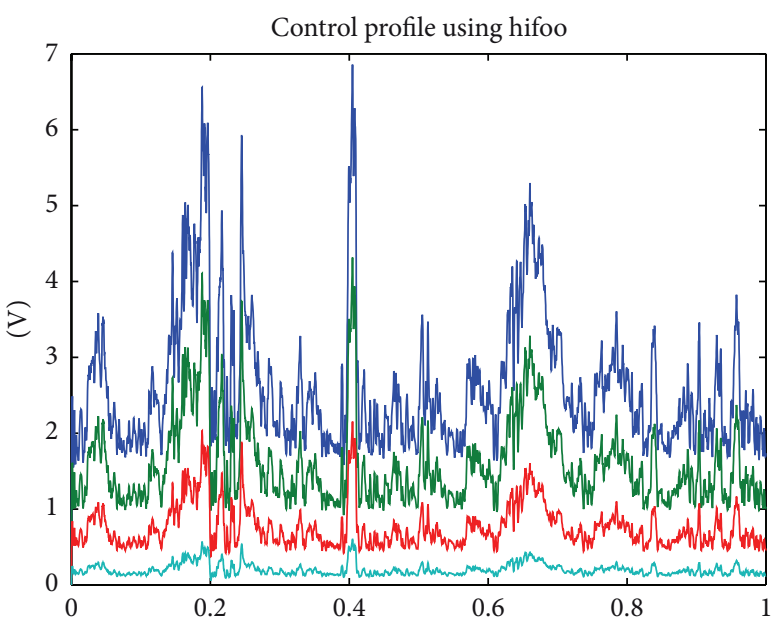

(s)

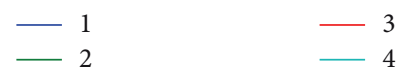

FIGURE 19: Stress at beam nodal points, using HIFOO.

is vibration reduction, while sustaining low steady state error, short recovery time, and small maximum uplift; at the same time, control energy must remain within operating limits.

The beam that was used was discretized using 1dimensional finite elements with two degrees of freedom per node. Piezoelectric actuators were embedded in it with the objective of reducing vibrations under deterministic as well as stochastic loading conditions.

Initially, we examined the linear quadratic control criterion using a reduced rank observer, which makes the simulation more realistic. To find the observer, we employed a robust pole location algorithm. By selecting appropriate weights, beam vibration reduction was achieved for stochastic loading cases. In all simulations, random noise has been introduced in measurements, so that the system better approximate reality, given that displacement measurement by means of 
piezoelectric sensors is not reliable. Next we applied more advanced control techniques, such as the $H_{\infty}$ criterion. The $H_{\infty}$ controller found is of order 36 .

In order to reduce computational requirements of the model, controller rank was reduced by means of nonparametric and nonconvex optimization, using the HIFOO controller. The controller exhibited good performance even for a significantly smaller system degree.

A natural consequence of the proposed research innovations is the acknowledgement of new scientific problems that can be used as the basis for further research beyond the scope of this work. The advantage of active control is the fact that it allows taking into account in the computation the worst case result of disturbances with uncertainty and system noise. Moreover, the active control can effectively cope with stronger input, permitting the design for a large frequency bandwidth. Results are noteworthy; vibration reduction is observed even for realistic wind loading, with piezoelectric component voltage kept within tolerance.

\section{Conflict of Interests}

The author declares that there is no conflict of interests regarding the publication of this paper.

\section{Acknowledgments}

The authors would like to thank the Wind Energy and Synthesis of Energy Systems Laboratory, of the Technological Educational Institute of Crete, for its offer of real life wind speed measurements in relevance with time that took place in Estavromenos of Heraclion, Crete.

\section{References}

[1] B. Bandyopadhyay, T. C. Manjunath, and M. Umapathy, Modeling, Control and Implementation of Smart Structures, vol. 350 of Lecture Notes in Control and Information Sciences, Springer, 2007.

[2] D. Halim, Vibration analysis and control of smart structures [Ph.D. thesis], 2002.

[3] B. Culshaw, "Smart structures-a concept or a reality?" Journal of Systems and Control Engineering, vol. 206, no. 1, pp. 1-8, 1992.

[4] S. S. Rao and M. Sunar, "Piezoelectricity and its use in disturbance sensing and control of flexible structures: a survey," Applied Mechanics Reviews, vol. 47, no. 4, pp. 113-123, 1994.

[5] T. Bailey and J. E. Ubbard Jr., "Distributed piezoelectricpolymer active vibration control of a cantilever beam," Journal of Guidance, Control, and Dynamics, vol. 8, no. 5, pp. 605-611, 1985.

[6] S. Hanagud, M. W. Obal, and A. J. Calise, "Optimal vibration control by the use of piezoceramic sensors and actuators," Journal of Guidance, Control, and Dynamics, vol. 15, no. 5, pp. 1199-1206, 1992.

[7] W.-S. Hwang and H. C. Park, "Finite element modeling of piezoelectric sensors and actuators," AIAA Journal, vol. 31, no. 5, pp. 930-937, 1993.
[8] B. Bona, M. Indri, and A. Tornambé, "Flexible piezoelectric structures-approximate motion equations and control algorithms," IEEE Transactions on Automatic Control, vol. 42, no. 1, pp. 94-101, 1997.

[9] W. Schiehlen and H. Schonerstedt, "Controller design for the active vibration damping of beam structure," in Proceedings of the Smart Mechanical Systems Adaptronics SAE International USA, pp. 1137-1146, 1998.

[10] S.-B. Choi, C.-C. Cheong, and C.-H. Lee, "Position tracking control of a smart flexible structure featuring a piezofilm actuator," Journal of Guidance, Control, and Dynamics, vol. 19, no. 6, pp. 1364-1369, 1996.

[11] F. Pourki, "Distributed controllers for flexible structures using piezoelectric actuators/sensors," in Proceedings of the 32nd IEEE Conference on Decision and Control (CDC '93), vol. 2, pp. 13671370, San Antonio, Tex, USA, December 1993.

[12] A. Benjeddou, M. A. Trindade, and R. Ohayon, "New shear actuated smart structure beam finite element," AIAA Journal, vol. 37, no. 3, pp. 378-383, 1999.

[13] X. D. Zhang and C. T. Sun, "Formulation of an adaptive sandwich beam," Smart Materials and Structures, vol. 5, no. 6, pp. 814-823, 1996.

[14] S. Raja, G. Prathap, and P. K. Sinha, "Active vibration control of composite sandwich beams with piezoelectric extensionbending and shear actuators," Smart Materials and Structures, vol. 11, no. 1, pp. 63-71, 2002.

[15] G. Zames and B. A. Francis, "Feedback, minimax sensitivity, and optimal robustness," IEEE Transactions on Automatic Control, vol. 28, no. 5, pp. 585-601, 1983.

[16] D. Halim and S. O. R. Moheimani, "Spatial resonant control of flexible structures-application to a piezoelectric laminate beam," IEEE Transactions on Control Systems Technology, vol. 9, no. 1, pp. 37-53, 2001.

[17] Z. Friedman and J. B. Kosmatka, "An improved two-node Timoshenko beam finite element," Computers and Structures, vol. 47, no. 3, pp. 473-481, 1993.

[18] G. E. Stavroulakis, G. Foutsitzi, E. Hadjigeorgiou, D. Marinova, and C. C. Baniotopoulos, "Design and robust optimal control of smart beams with application on vibrations suppression," Advances in Engineering Software, vol. 36, no. 11-12, pp. 806-813, 2005.

[19] B. Miara, G. Stavroulakis, and V. Valente, Topics on Mathematics for Smart Systems: Proceedings of the European Conference, Rome, Italy, 26-28 October 2006, World Scientific, Singapore, 2007.

[20] A. Moutsopoulou, A. Pouliezos, and G. E. Stavroulakis, "Modelling with uncertainty and robust control of smart beams," in Proceedings of the 9th International Conference on Computational Structures Technology (CST '09), B. H. V. Topping and M. Papadrakakis, Eds., paper 35, Civil Comp Press, Athens, Greece, September 2008.

[21] H. F. Tiersten, Linear Piezoelectric Plate Vibrations, Plenum Press, New York, NY, USA, 1st edition, 1969.

[22] S. M. Yang and Y. J. Lee, "Optimization of noncollocated sensor/actuator location and feedback gain in control systems," Smart Materials and Structures, vol. 2, no. 2, pp. 96-102, 1993.

[23] O. H. Bosgra and H. Kwakernaak, Design Methods for Control Systems, Course Notes, Dutch Institute for Systems and Control, 2001.

[24] H. Kwakernaak, "Robust control and $H_{\infty}$-optimizationtutorial paper," Automatica, vol. 29, no. 2, pp. 255-273, 1993. 
[25] R. C. Ward, "Balancing the generalized eigenvalue problem," SIAM Journal on Scientific and Statistical Computing, vol. 2, no. 2, pp. 141-152, 1981.

[26] A. L. Tits and Y. Yang, "Globally convergent algorithms for robust pole assignment by state feedback," IEEE Transactions on Automatic Control, vol. 41, no. 10, pp. 1432-1452, 1996.

[27] H. Kimura, "Robust stabilizability for a class of transfer functions," IEEE Transactions on Automatic Control, vol. 29, no. 9, pp. 788-793, 1984.

[28] B. A. Francis, A Course on $H_{\infty}$ Control Theory, Lecture Notes in Control and Information Sciences, Springer, 1987.

[29] J. C. Doyle, K. Glover, P. P. Khargonekar, and B. A. Francis, "State-space solutions to standard $\mathrm{H}_{2}$ and $H_{\infty}$ control problems," IEEE Transactions on Automatic Control, vol. 34, no. 8, pp. 831-847, 1989.

[30] A. Packard, J. Doyle, and G. Balas, "Linear, multivariable robust control with a $\mu$ perspective," Journal of Dynamic Systems, Measurement and Control, vol. 115, no. 2, pp. 426-438, 1993.

[31] J. V. Burke, D. Henrion, A. S. Lewis, and M. L. Overton, "HIFOO-a MATLAB package for fixed-order controller design and $H_{\infty}$ optimization," in Proceedings of the 5th IFAC Symposium on Robust Control Design (ROCOND '06), pp. 339344, Toulouse, France, July 2006.

[32] J. V. Burke, D. Henrion, A. S. Lewis, and M. L. Overton, "Stabilization via nonsmooth, nonconvex optimization," IEEE Transactions on Automatic Control, vol. 51, no. 11, pp. 1760-1769, 2006.

[33] J. V. Burke, A. S. Lewis, and M. L. Overton, "A robust gradient sampling algorithm for nonsmooth, nonconvex optimization," SIAM Journal on Optimization, vol. 15, no. 3, pp. 751-779, 2005.

[34] J. V. Burke and M. L. Overton, "Variational analysis of nonLipschitz spectral functions," Mathematical Programming B, vol. 90, no. 2, pp. 317-351, 2001.

[35] D. Hinrichsen and A. J. Pritchard, Mathematical Systems Theory I: Modelling, State Space Analysis, Stability and Robustness, vol. 48 of Texts in Applied Mathematics, Springer, 2006.

[36] M. Millston, HIFOO 1.5: structured control of linear systems with a non trivial feedthrough [M.S. thesis], Courant Institute of Mathematical Sciences, New York University, New York, NY, USA, 2006. 


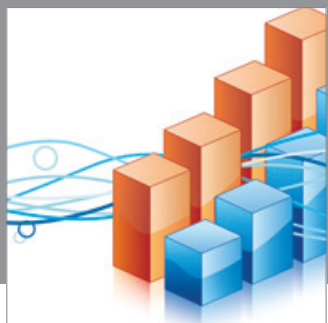

Advances in

Operations Research

mansans

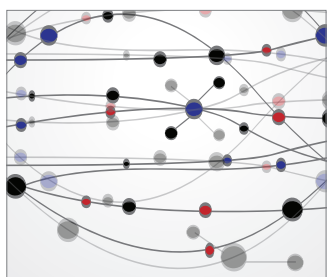

The Scientific World Journal
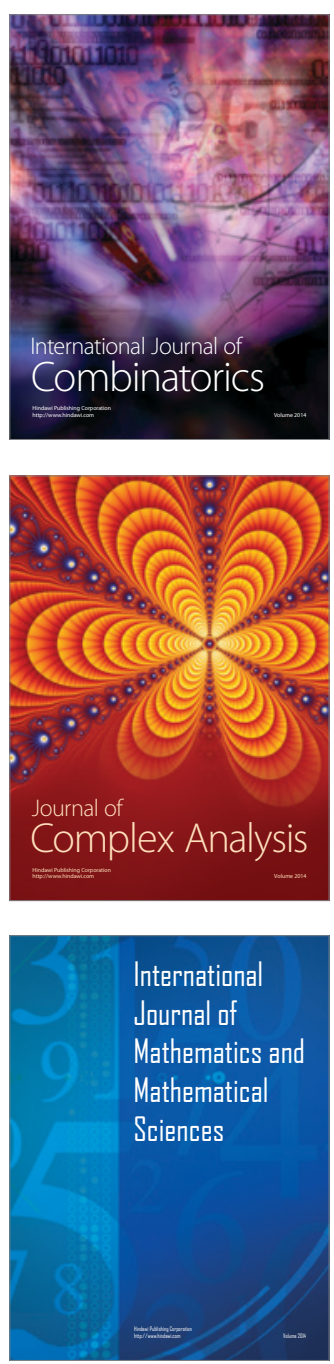
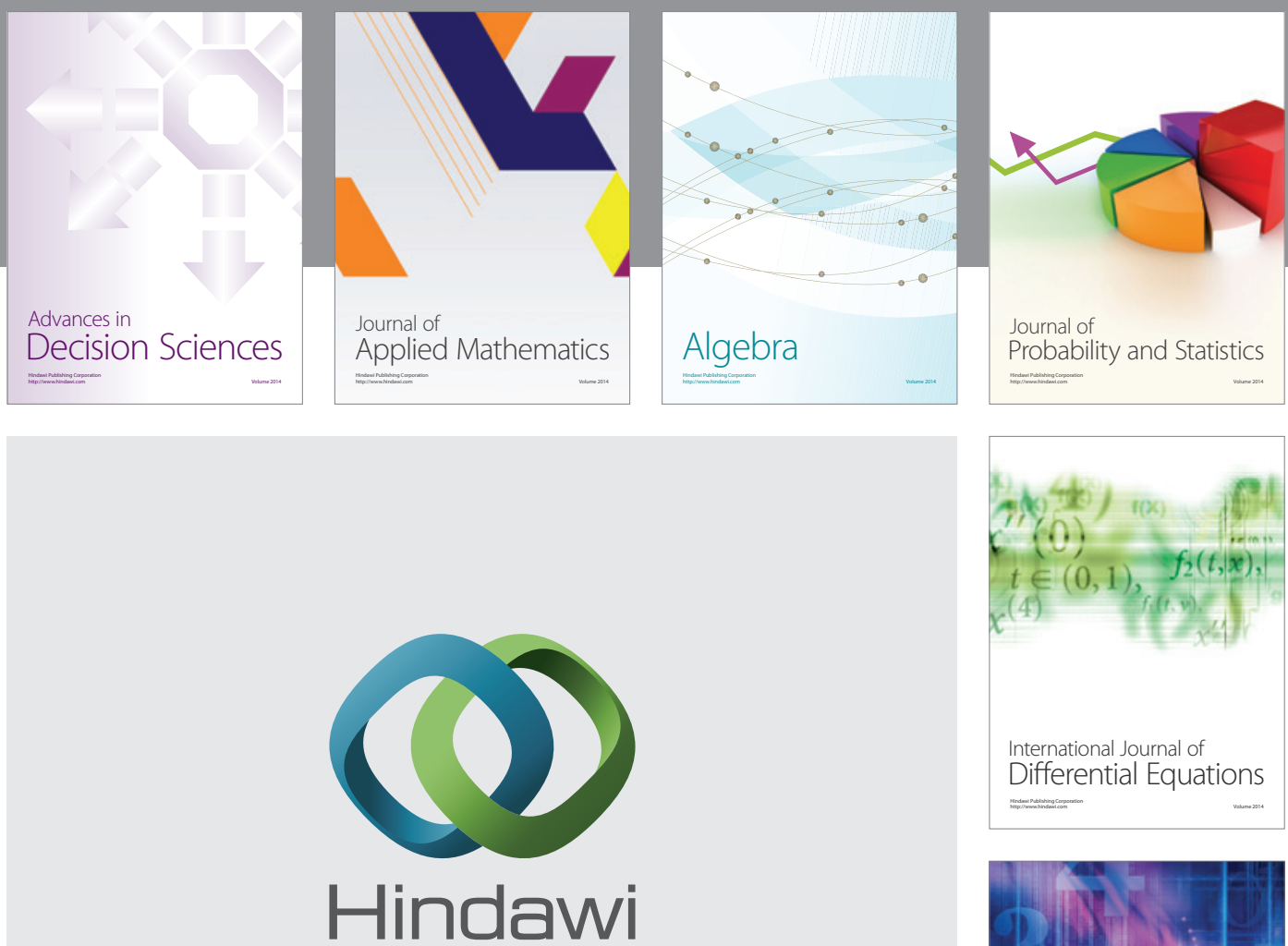

Submit your manuscripts at http://www.hindawi.com
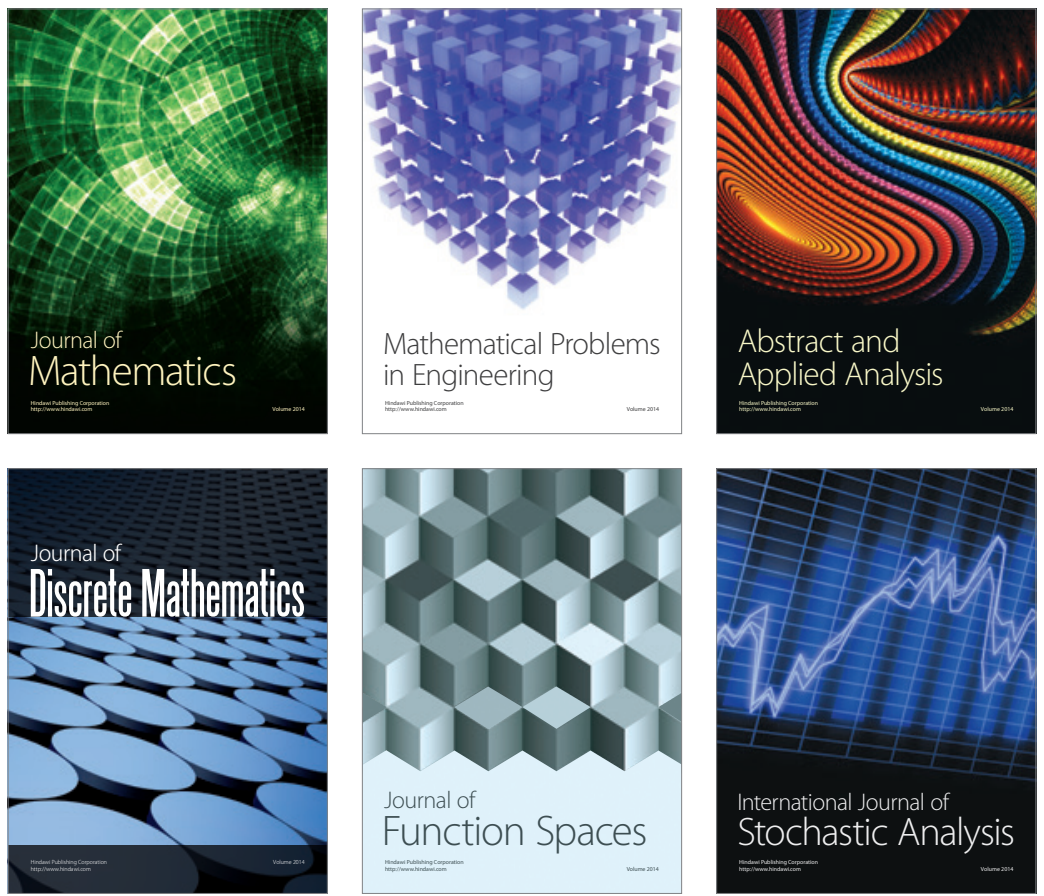

Journal of

Function Spaces

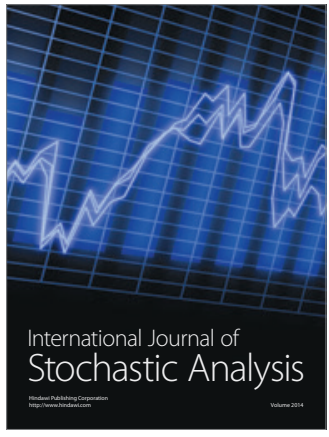

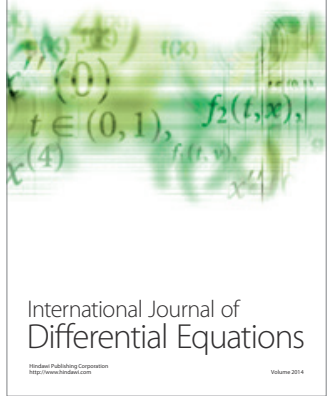
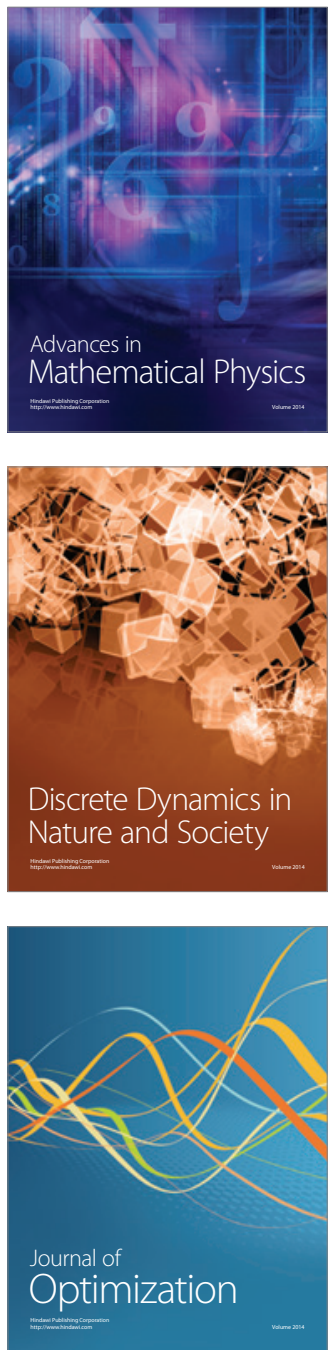